\title{
High-resolution transmission electron microscopy on $\mathrm{KH}_{x}-\mathrm{GIC}$ 's
}

\author{
L. Salamanca-Riba, ${ }^{\text {a) }}$ N.-C. Yeh, and M. S. Dresselhaus \\ Department of Physics, Massachusetts Institute of Technology, Cambridge, Massachusetts 02139 \\ M. Endo \\ Shinshu University, Nagano-shi 380, Japan \\ T. Enoki \\ Institute for Molecular Science, Okazaki 444, Japan
}

(Received 5 August 1985; accepted 31 October 1985)

\begin{abstract}
The in-plane and $c$-axis structure of $\mathrm{KH}_{x}-\mathrm{GIC}$ 's and $\mathrm{KD}_{y}-\mathrm{GIC}$ 's is studied using transmission electron microscopy (TEM) and x-ray diffraction as a function of intercalation temperature and time. With the TEM, two commensurate in-plane phases are found to coexist in these compounds with relative concentrations depending on intercalation conditions. When the direct intercalation method is used, the first step of intercalation is the formation of a stage $n$ potassium-GIC and the final compound is a stage $n \mathrm{KH}_{x}-\mathrm{GIC}$ (or $\mathrm{KD}_{y}-\mathrm{GIC}$ ). Highresolution $(00 l)$ lattice images show direct evidence for intermediate phases in the intercalation process. These intermediate phases are hydrogen (deuterium) deficient and are found at the boundary between pure potassium regions and regions with high hydrogen (deuterium) content. A comparison of the structure for the two methods of intercalation of $\mathrm{KH}$ is also presented.
\end{abstract}

\section{INTRODUCTION}

High-resolution transmission electron microscopy (TEM) provides information about the structure of materials at a microscopic level that cannot be obtained by other methods such as x-ray diffraction, Raman scattering spectroscopy, neutron scattering, etc. In this work we apply the TEM technique to study the structure and process of intercalation of $\mathrm{KH}$ and $\mathrm{KD}$ into graphite. Complementary $\mathrm{x}$-ray measurements are also reported.

Graphite intercalation compounds (GIC's) are prepared by the insertion of layers of foreign species between the graphite layers. In these compounds, $n$ (the stage index) graphite layers separate two neighboring intercalate layers. ${ }^{1}$ In addition to the superlattice structure perpendicular to the layer planes, some of the intercalants form in-plane superlattices that are commensurate with the graphite lattice. Often several phases are found to coexist in the same sample. In general, the method used to synthesize GIC's depends on the intercalant and on the desired stage.

Synthesis of $\mathrm{KH}_{x}-\mathrm{GIC}$ 's or $\mathrm{KD}_{y}-\mathrm{GIC}$ 's can be achieved by direct intercalation ${ }^{2}$ of $\mathrm{KH}(\mathrm{KD})$ or by chemical absorption ${ }^{3}$ of hydrogen (deuterium) into potassium-GIC's. However, stage $1 \mathbf{K H}_{x}-\left(\mathrm{KD}_{y}\right)-\mathrm{GIC}$ 's have only been prepared by the direct intercalation of KH (KD). For the stage 2 compound which can be prepared by either technique, the stoichiometry of the compound depends on the intercalation process, with a higher hydrogen (deuterium) uptake for the direct intercalation $^{2}$ of $\mathrm{KH}_{x}\left(\mathrm{KD}_{y}\right) \quad[x(y) \leqslant 0.8]$ than for the

\footnotetext{
a) Present address: General Motors Research Lab, Warren, Michigan 48090-9055.
}

chemical absorption ${ }^{4}$ of hydrogen (deuterium) into stage 1 potassium-GIC $[x(y) \leqslant 0.66]$. The saturation value of $x$ for stage $1 \mathrm{KH}_{x}-\mathrm{GIC}$ 's is $x \approx 0.8$ (see Ref. 2 ). In this work we used high-resolution transmission electron microscopy (TEM) to study and compare the structure of $\mathrm{KH}_{x}-\mathrm{GIC}$ 's (or $\mathrm{KD}_{y}-\mathrm{GIC}$ 's) obtained by the two intercalation methods. We have found that both $\mathrm{KH}$ and KD form two commensurate in-plane phases: a $(2 \times 2) R 0^{\circ}$ and a $(\sqrt{3} \times \sqrt{3}) R 30^{\circ}$ phase which are dominant in different intercalation temperature ranges. We have used highly oriented pyrolytic graphite (HOPG), kish single crystal graphite, and benzene-derived graphite fibers (BDGF) as host materials. The HOPG and kish-based samples give information about both the inplane and $c$-axis structure, whereas the fiber host provides information predominantly about the $c$-axis structure. We present here studies on the structure of these compounds as well as the dependence of the structure on stage and intercalation temperature and time. During the TEM observation, electron beam-induced desorption of the hydrogen takes place, thereby allowing detailed examination of this desorption process.

\section{EXPERIMENTAL DETAILS}

The samples used in this experiment were intercalated using either the direct intercalation method ${ }^{2,5}$ or the chemical absorption method. ${ }^{3,4}$ After completion of the intercalation process, the samples based on HOPG and kish single crystal graphite were characterized for stage using $(00 l) \mathrm{x}$-ray diffraction through a glass ampoule containing hydrogen gas and some intercalant powder. The samples based on BDGF were characterized for stage using the TEM. 
The TEM observation ${ }^{6}$ was done using two transmission electron microscopes. A JEOL $200 \mathrm{CX}$ (having a spherical aberration coefficient $\mathrm{C}_{s}=2.8 \mathrm{~mm}$ ) with a $\mathrm{LaB}_{6}$ filament was used to obtain the high-resolution images, and a JEM $100 \mathrm{CX}$ with a pointed tungsten filament was used for the low magnification studies. The samples for electron microscopy were prepared inside a glove bag under an argon atmosphere. The HOPGbased samples were prepared by repeated cleavage until a sample with thin regions $(\leqslant 300 \AA)$ along the edges was obtained. The thin sample was placed between $\mathrm{Cu}$ 400 mesh electron microscope grids. The intercalated fibers, on the other hand, were directly placed between the $\mathrm{Cu}$ grids with no special thinning technique. The grids containing the samples were put in the sample holder which was then taken out of the glove bag and quickly introduced into the microscope column, to minimize the exposure to air.

In studying the structure of $\mathrm{KH}_{x}$-GIC's (and $\mathrm{KD}_{y}$-GIC's) using the TEM, we have obtained information from $(h k 0)$ and $(h k l)$ electron diffraction patterns, dark field images and high-resolution lattice images. The dark field images were obtained by tilting the beam until the desired reflection was on the optic axis of the microscope. ${ }^{6}$ An objective aperture was then placed at the back focal plane of the microscope, including only the desired reflection. The high-resolution lattice images were obtained under axial illumination by placing an objective aperture that encompassed the unscattered beam and reflections up to $1.70 \AA^{-1}$ for the in-plane images and $1.88 \AA^{-1}$ for the $c$-axis lattice images. The interplanar spacings were determined from optical diffractograms obtained from the negatives of the images. ${ }^{7}$

\section{RESULTS AND DISCUSSION}

Pristine KH and KD have essentially identical crystal structures. Both compounds form fcc lattices with a lattice parameter of $5.70 \AA$. Therefore, similar structures are expected for both intercalated compounds, as was verified in the present work. We present below specific results for the $\mathrm{KH}_{x}-\mathrm{GIC}$ 's.

The intercalation process of $\mathrm{KH}(\mathrm{KD})$ into graphite was studied using $(00 l) \mathrm{x}$-ray diffraction on HOPG samples intercalated at several temperatures $T_{i}$ and for various times $t_{i}$ (see Ref. 8). The x-ray diffraction results showed that for $350^{\circ} \mathrm{C}<T_{i}<430{ }^{\circ} \mathrm{C}$, the first step of intercalation (after a few hours) was a stage 1 potassium-GIC (repeat distance $I_{c}=5.35 \pm 0.03 \AA$ ) though $\mathrm{KH}$ and not $\mathrm{K}$ was present in the ampoule. After a few days of intercalation, peaks in the $(00 l) \mathrm{x}$-ray diffractograms corresponding to a mixture of stage 1 potassium-GIC and stage $1 \mathrm{KH}_{x}-\mathrm{GIC}\left(I_{c}=8.55 \pm 0.03\right.$ $\AA$ ) were obtained with admixtures of stage $2 \mathrm{KH}_{x}$-GIC that decreased with time. Finally, after 10 days of intercalation, only peaks corresponding to stage $1 \mathrm{KH}_{x}-$
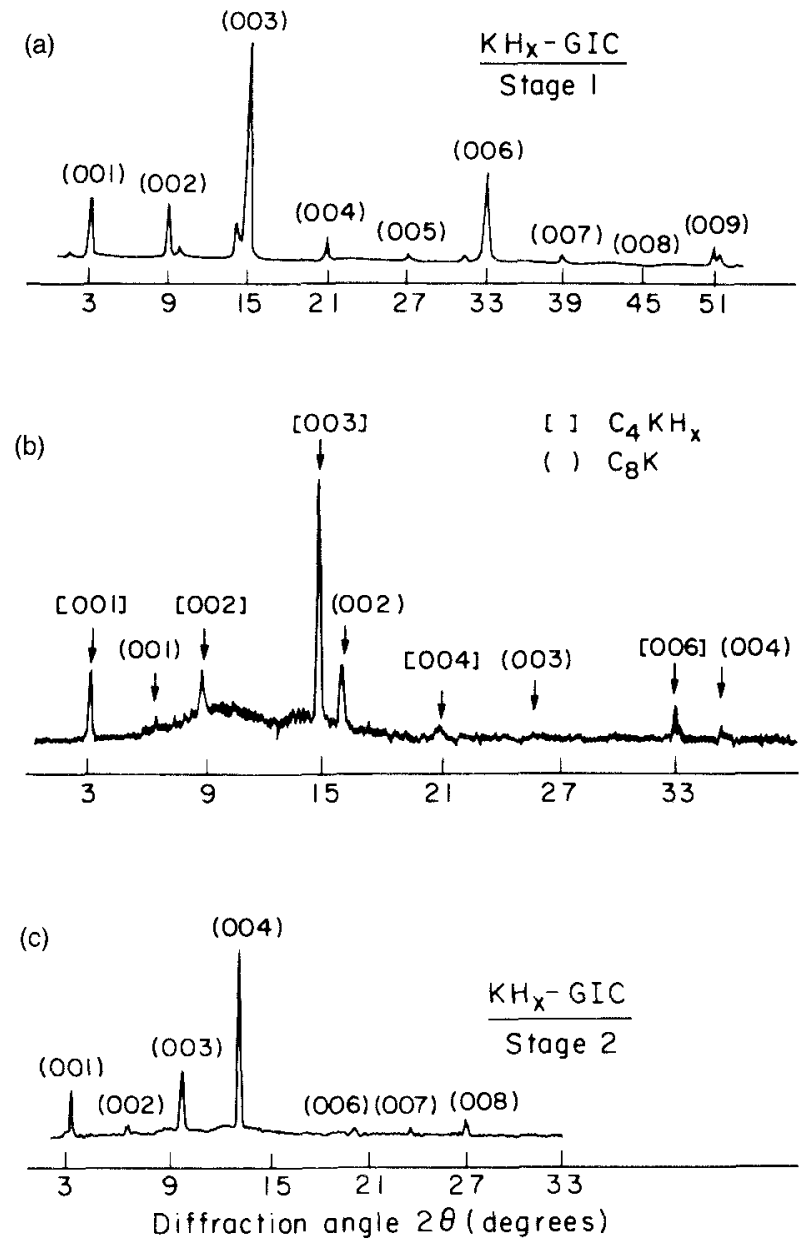

FIG. 1. The ( $00 l) \mathrm{x}$-ray diffractograms of (a) a stage 1 sample intercalated with $\mathrm{KH}$ at $430^{\circ} \mathrm{C}$, (b) a sample intercalated with $\mathrm{KH}$ at $290^{\circ} \mathrm{C}$ for 10 days showing a mixture of stage 1 potassium-GIC and stage 1 $\mathbf{K H}_{x}-$ GIC, and (c) a stage $2 \mathrm{KH}_{x}$ - GIC sample, 1 year subsequent to intercalation. The repeat distances are: (a) $8.55 \AA\left(\mathrm{C}_{4} \mathrm{KH}_{x}\right)$ and $12.08 \AA\left(\mathrm{C}_{8} \mathrm{KH}_{x}\right)$, (b) $8.55 \AA\left(\mathrm{C}_{4} \mathrm{KH}_{x}\right)$ and $5.35 \AA\left(\mathrm{C}_{8} \mathrm{~K}\right)$, and (c) $11.93 \AA\left(\mathrm{C}_{8} \mathrm{KH}_{x}\right)$. The chemical formulas for the stage $1 \mathrm{~K}-\mathrm{GIC}$ and the stage 1 and stage $2 \mathbf{K H}_{x}-$ GIC's are taken from Ref. 2.

GIC and a small admixture of stage $2 \mathrm{KH}_{x}$-GIC were observed. The $(00 l) \mathrm{x}$-ray diffractogram for a stage 1 sample after 10 days of intercalation is shown in Fig. 1(a). This intercalation process was also observed by Guérard et al. ${ }^{9}$ Mixtures of stage $1 \mathrm{KH}_{x}-$ GIC and stage 1 potassium-GIC were obtained for intercalation temperatures in the range $250^{\circ} \mathrm{C}-350^{\circ} \mathrm{C}$ even after intercalation times of 10 days. Figure $1(\mathrm{~b})$ shows the $(00 l) \mathrm{x}$ ray diffractogram of a sample intercalated with $\mathrm{KH}$ at $290^{\circ} \mathrm{C}$. For $210^{\circ} \mathrm{C}<T_{i}<350^{\circ} \mathrm{C}$, mixed stages (potassium-GIC and $\mathrm{KH}_{x}-\mathrm{GIC}$ ) were obtained. It has been reported ${ }^{4}$ that stage 2 can be obtained using the direct intercalation method for temperatures between $250^{\circ} \mathrm{C}$ $300^{\circ} \mathrm{C}$. Up to now we have not been able to obtain pure stage $2 \mathrm{KH}_{x}$-GIC samples for intercalation temperatures in this range. On the other hand, single stage 2 $\mathrm{KH}_{x}$-GIC samples have been obtained for tempera- 

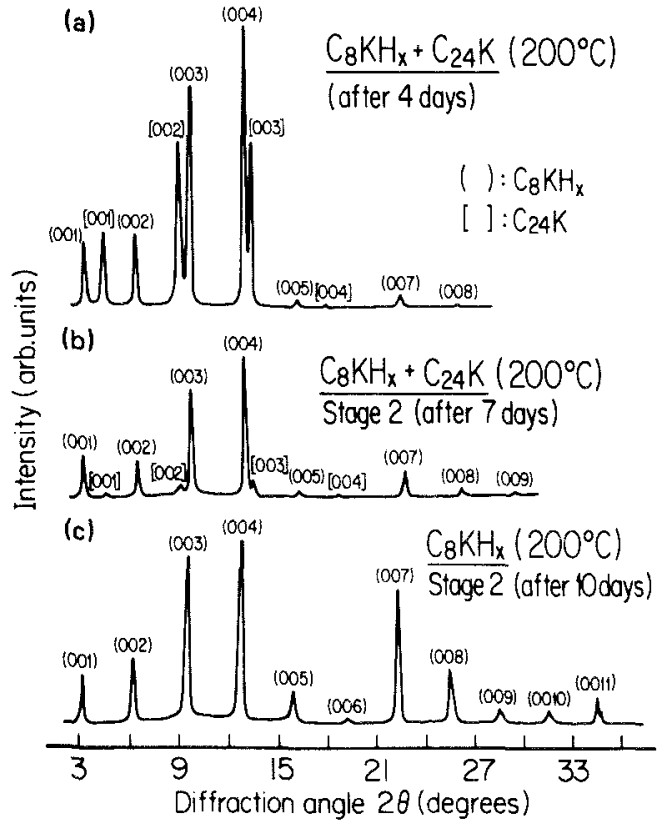

FIG. 2. (a)-(c) The $(00 l)$ x-ray diffractograms of samples intercalated with $\mathrm{KH}$ at $200^{\circ} \mathrm{C}$ showing the intercalation process as a function of intercalation time. The repeat distances are: $8.75 \AA\left(\mathrm{C}_{24} \mathrm{~K}\right)$ and $12.08 \AA\left(\mathrm{C}_{8} \mathrm{KH}_{x}\right)$ (see text). The chemical formulas for the stage 2 $\mathrm{K}-\mathrm{GIC}$ and stage $2 \mathrm{KH}_{x}-\mathrm{GIC}$ are taken from Ref. 2 and $\mathrm{C}_{8} \mathrm{KH}_{x}+\mathrm{C}_{24} \mathrm{~K}$ denotes a mixture of stage $2 \mathrm{KH}_{x}-\mathrm{GIC}$ and stage 2 K-GIC. tures in the range $200^{\circ} \mathrm{C}<T_{i}<210^{\circ} \mathrm{C}$ as shown below.

For the stage 2 compounds (see Fig. 2), the first step of intercalation was stage 2 potassium-GIC $\left(I_{c}=8.75 \pm 0.03 \AA\right)$, and the final compound (after $\sim 10$ days) that was obtained was a stage $2 \mathrm{KH}_{x}$-GIC $\left(I_{c}=12.08 \pm 0.03 \AA\right)$ [see Fig. 2(c) ]. The repeat distance of $12.08 \AA$ obtained for stage $2 \mathrm{KH}_{x}-\mathrm{GIC}$ is larger than that obtained by Doll et al. ${ }^{10}(11.87 \pm 0.02 \AA)$ and by Guérard et $a l^{2}{ }^{2}(11.88 \AA)$. Reflectivity measurements performed on the sample with repeat distance of $11.87 \AA$ suggested that the sample contained low hydrogen content. ${ }^{10}$ A repeat distance of $11.93 \AA$ [shown in Fig. 1 (c) ] was obtained using $x$-ray diffraction from samples that had been intercalated a year before. ${ }^{11}$ It is interesting to note that essentially the same Shubnikovde Haas frequencies were obtained from the samples with a smaller repeat distance of $11.93 \AA$ as those with a larger repeat distance of $12.08 \AA .{ }^{11}$ It is possible that the two different repeat distances correspond to a different hydrogen content in the intercalate layer, with the smaller hydrogen content corresponding to the smaller repeat distance. There is also the possibility (as explained below) that some $\mathrm{KH}$ (in its pristine form) is contained in inclusions in the intercalate layer of the samples with larger repeat distance, as was found for the case of $\mathrm{NaH}-\mathrm{GIC}$ 's. ${ }^{9}$

The process of intercalation described above is dif-

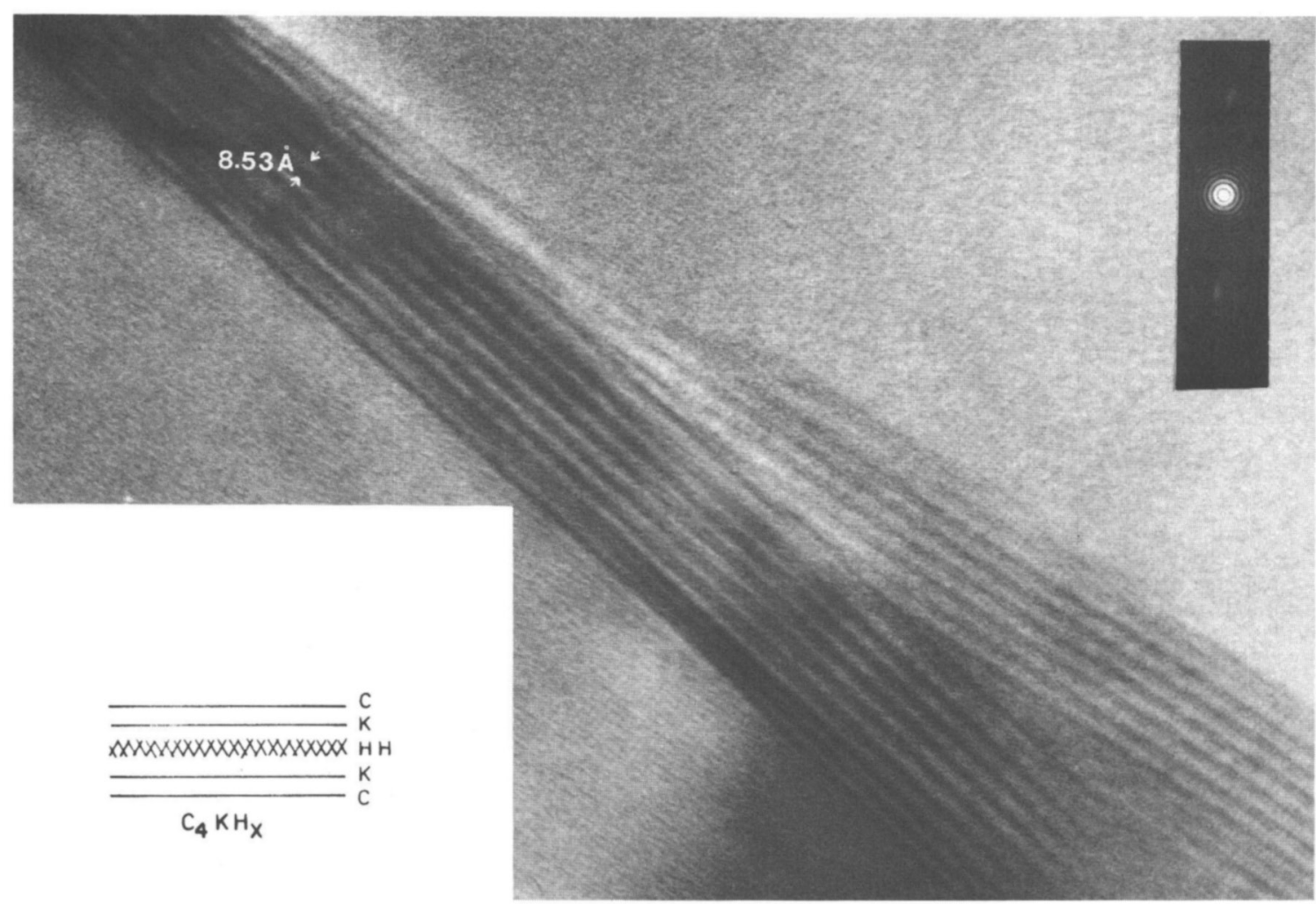

FIG. 3. The $c$-axis lattice image of a stage $1 \mathrm{KH}_{x}$-GIC sample intercalated into HOPG at $430^{\circ} \mathrm{C}$. The insets are a schematic diagram of the structure along the $c$ axis and an optical diffractogram taken from the negative of the photograph. 
ferent from that observed for the chemical absorption of hydrogen into stage 1 potassium. ${ }^{4}$ In the chemical absorption method, the first step of intercalation is stage 1 potassium-GIC (obtained by intercalation of potassium only), and the final compound (after the absorption of hydrogen) is a stage $2 \mathrm{KH}_{x}-\mathrm{GIC}^{4}$ Some of the samples used in the $\mathrm{x}$-ray diffraction experiment were used to prepare samples for the TEM experiment. The TEM results ${ }^{6}$ were consistent with the $x$-ray diffraction results, ${ }^{8}$ as explained below.

The $c$-axis repeat distance $I_{c}$ was also deduced using the TEM from $(00 l)$ lattice fringes obtained either from regions of the intercalated HOPG samples that were bent in such a way that the $(00 l)$ planes were parallel to the electron beam direction or from the edges of the intercalated BDGF. Figure 3 shows a $c$-axis lattice image (a view normal to the $c$ axis) of an HOPG sample intercalated at $430^{\circ} \mathrm{C}$. This figure shows a single staged sample with $I_{c}=8.53 \pm 0.08 \AA$. The same repeat distance (within experimental error) of $8.55 \AA$ was obtained from $(00 l) \mathrm{x}$-ray diffraction from the same stage 1 sample. This repeat distance is in agreement with the sandwich model suggested by Guérard, ${ }^{4}$ and schematically represented in the inset to Fig. 3. In this model the intercalate layer forms a three-layer sandwich along the $c$ axis with two layers of $\mathrm{K}$ atoms, one above and one below a layer of $\mathrm{H}$ atoms. This structure is similar to that of KHg-GIC's, ${ }^{12-14}$ where the intercalate layer forms a three-layer sandwich with the $\mathrm{Hg}$ layer sandwiched between the two layers of $\mathrm{K}$ atoms. It has been suggested that the $\mathrm{Hg}$ atoms are arranged in two staggered layers of $\mathrm{Hg}$, with a separation between the $\mathrm{Hg}$ layers of $\sim 0.254 \AA$ (see Refs. 13 and 14). Evidence is given below that the in-plane structure of $\mathrm{KH}_{x}$-GIC's is also similar to that of $\mathrm{KHg}-\mathrm{GIC}$ 's.

The $(00 l)$ lattice images of $\mathrm{KH}_{x}-\mathrm{GIC}$ samples intercalated either at lower temperatures or for shorter times showed several repeat distances and these results gave insight into the intercalation process. Figure 4 shows $c$-axis lattice images of a sample intercalated with $\mathrm{KH}$ at $210^{\circ} \mathrm{C}$ for 8 days. This figure shows three regions with repeat distances of $8.80 \pm 0.08 \AA, 10.62 \pm 0.08 \AA$, and $11.90 \pm 0.08 \AA$. In the three regions, fringes with a separation of $\sim 3.35 \AA$ are periodically stacked between layered structures with spacings $5.45 \AA, 7.27 \AA$, and $8.55 \AA$ for the three regions (see Fig. 4). The fringes separated by $3.35 \AA$ are identified with pairs of graphite layers, thus indicating that all three regions correspond to stage 2 compounds. The values of $8.80 \AA$ and $11.90 \AA$ correspond to a stage 2 potassium-GIC and a stage 2 $\mathrm{KH}_{x}-\mathrm{GIC}$, respectively. The value of $10.62 \AA$ can be explained as the repeat distance of a stage 2 hydrogen deficient region which separates a region of a pure stage 2 potassium-GIC from a region of a stage $2 \mathrm{KH}_{x}-$ GIC with normal hydrogen concentration. The three regions are presented schematically in the figure. This result is in agreement with the $\mathrm{x}$-ray diffraction result, where it was found that for $t_{i}<10$ days, a mixture of stage 2 potassium-GIC and stage $2 \mathrm{KH}_{x}-\mathrm{GIC}$ was obtained for $200^{\circ} \mathrm{C}<T_{i}<210^{\circ} \mathrm{C}$ (see Fig. 2). It is possible that in the $\mathrm{KH}_{x}$-GIC's with $x \sim 0.8$ (the saturation limit), the hydrogen atoms within the intercalate sandwich are staggered from each other with respect to the $c$ axis, as is the case proposed for the KHg-GIC's. ${ }^{13,14}$ Thus it is

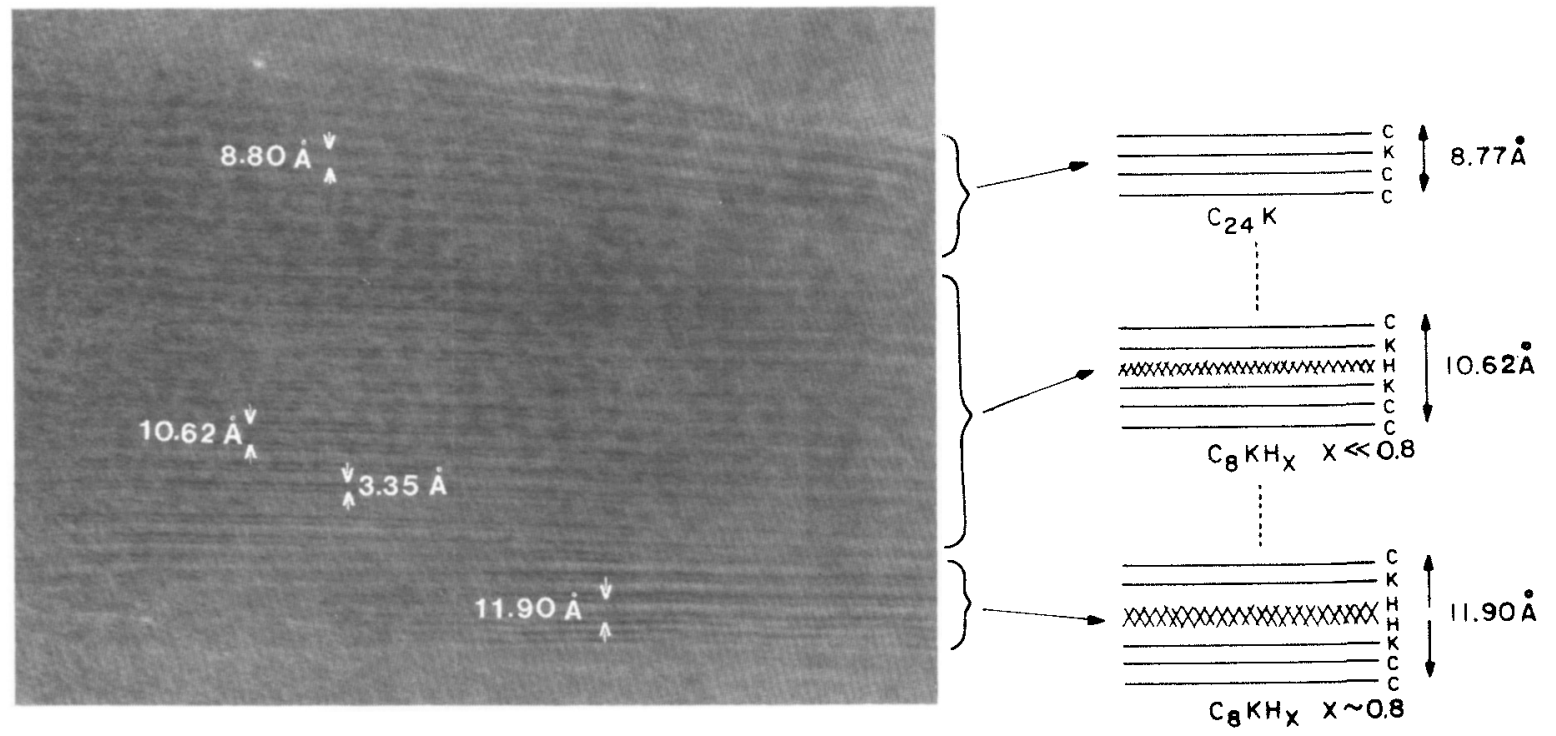

FIG. 4. The $c$-axis lattice image of a stage $2 \mathrm{C}_{24} \mathrm{~K} / \mathrm{C}_{8} \mathrm{KH}_{x}$ sample prepared by direct intercalation with $\mathrm{KH}$ at $210{ }^{\circ} \mathrm{C}$. The schematic shows model structures for the stage $2 \mathrm{~K}$-GIC, for a hydrogen deficient boundary region and for the stage $2 \mathrm{KH}_{x}-$ GIC. The value of $8.77 \AA$ for the stage 2 potassium was reported in Ref. 1. The chemical formulas for the stage $2 \mathrm{~K}-\mathrm{GIC}$ and stage $2 \mathrm{KH}_{x}-\mathrm{GIC}$ are taken from Ref. 2. 


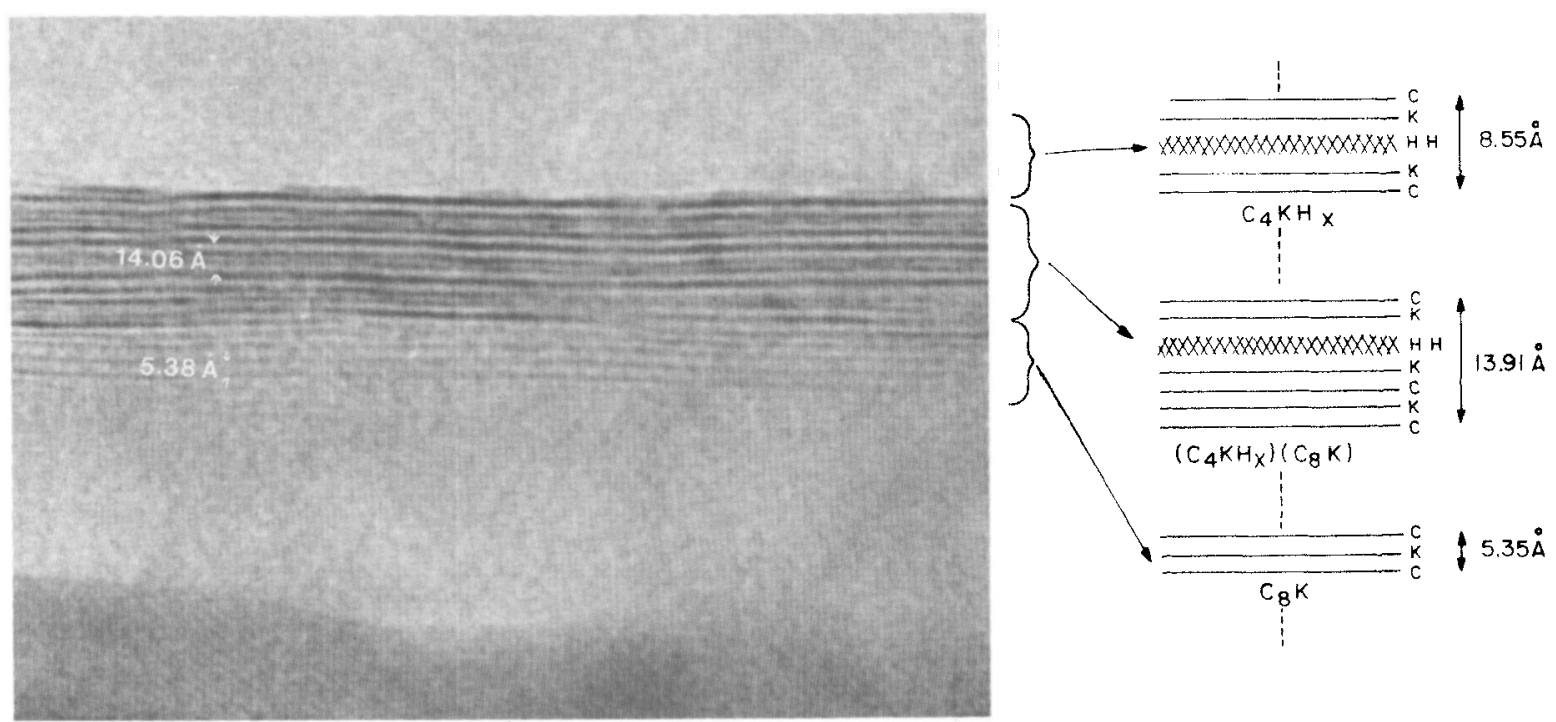

FIG. 5. The $c$-axis lattice image of a stage 1 sample of $\mathrm{C}_{8} \mathrm{~K} / \mathrm{C}_{4} \mathrm{KH}_{x}$ prepared by the direct intercalation of $\mathrm{KH}$ at $290^{\circ} \mathrm{C}$. The schematic shows model structures for the stage $1 \mathrm{~K}$-GIC, for the stage $1 \mathrm{KH}_{x}-\mathrm{GIC}$ and for a superlattice structure proposed for the hydrogen-deficient region at the boundary between the $\mathrm{C}_{8} \mathrm{~K}$ region and the $\mathrm{C}_{4} \mathrm{KH}_{x}$ region. The chemical formulas for the stage $1 \mathrm{~K}-\mathrm{GIC}$ and stage $1 \mathrm{KH}{ }_{x}-\mathrm{GIC}$ are taken from Ref. 2.

possible that a single value for the repeat distance is obtained for the hydrogen deficient boundary regions (between a pure potassium intercalated region and a region containing some hydrogen) because for low hydrogen concentrations, a single planar layer of hydrogen atoms within the intercalate sandwich may be formed; the hydrogen deficient boundary region denotes the region between a pure potassium intercalated region and a region containing hydrogen in the saturation limit.

Figure 5 shows a $c$-axis lattice image of an HOPG sample intercalated with $\mathrm{KH}$ at $290^{\circ} \mathrm{C}$. Analysis of the $(00 l)$ x-ray diffractograms obtained from this sample showed two repeat distances of $5.35 \pm 0.03 \AA$ and $8.55 \pm 0.03 \AA$ (see inset to Fig. 5) suggesting an admixture of a stage 1 potassium-GIC and a stage $1 \mathrm{KH}_{\mathbf{x}}$ GIC. The $c$-axis lattice image in Fig. 5 taken with the TEM, however, shows two repeat distances of $5.38 \pm 0.08 \AA$ and $14.06 \pm 0.08 \AA$. The repeat distance of $8.55 \pm 0.08 \AA$ was also found in other regions of the sample. Similar values for the repeat distances were obtained from $(00 l)$ lattice fringe images of benzene-derived graphite fibers intercalated with $\mathrm{KH}$ at $320^{\circ} \mathrm{C}$. The $I_{c}$ of $5.38 \AA$ corresponds to a stage 1 potassiumGIC. The repeat distance of $14.06 \AA$ can be related to a hydrogen-deficient region in the boundary between a stage 1 potassium-GIC region and a stage $1 \mathrm{KH}_{\mathbf{x}}-\mathrm{GIC}$ region. This hydrogen deficient region in the boundary can be interpreted as being formed by a periodic mixture of alternating layers of stage one potassium-GIC and stage $1 \mathrm{KH}-\mathrm{GIC}$ as shown schematically in the inset to Fig. 5, where $5.35 \AA+8.55 \AA \rightarrow 13.91 \AA$. The fact that the repeat distance of $14.06 \AA$ corresponds to boundary regions was corroborated by $c$-axis lattice images obtained from other regions of this sample, which showed the repeat distances of $14.06 \AA$ and $8.55 \AA$. This result is also in agreement with the $\mathrm{x}$-ray diffraction result ${ }^{8}$ where a mixture of stage 1 potassium-GIC and stage 1 $\mathrm{KH}_{x}-\mathrm{GIC}$ was obtained for $290^{\circ} \mathrm{C}<T_{i}<350^{\circ} \mathrm{C}$ even for intercalation times up to 10 days [see Fig. 1(b)].

A repeat distance of $13.80 \AA$ has been observed for stage $2 \mathbf{K H}_{x}-$ GIC samples intercalated by the chemical absorption of hydrogen into a stage 1 potassium-GIC that were encapsulated with $\mathrm{K}$ metal and heated to a temperature just above the melting point of the potassium metal. ${ }^{4}$ The repeat distance of $13.80 \AA$ was also interpreted ${ }^{4}$ as a periodic mixture of a stage 1 $\mathrm{KH}_{x}-\mathrm{GIC}$ and a stage 1 potassium-GIC. The smaller repeat distance of $13.80 \AA$ compared to $14.06 \AA$ is probably due to the fact that the hydrogen content in a $\mathrm{KH}_{x}-\mathrm{GIC}$ is lower when the chemical absorption method is used than when the direct intercalation method is used.

When the direct intercalation method is used, the repeat distances of $14.06 \AA(n=1)$ and $10.62 \AA$ $(n=2)$ at the boundary between a pure potassium-GIC region and a hydrogen saturated region are observed in small regions ( $\sim 100 \AA$ thick $)$ of the sample. Consequently, such small regions are less likely to be observed using $\mathrm{x}$-ray diffraction, since $\mathrm{x}$-ray diffraction is a bulk probe. Thus the TEM results support the $x$-ray diffraction results for the process of intercalation of $\mathrm{KH}$ into graphite and give additional information about the 


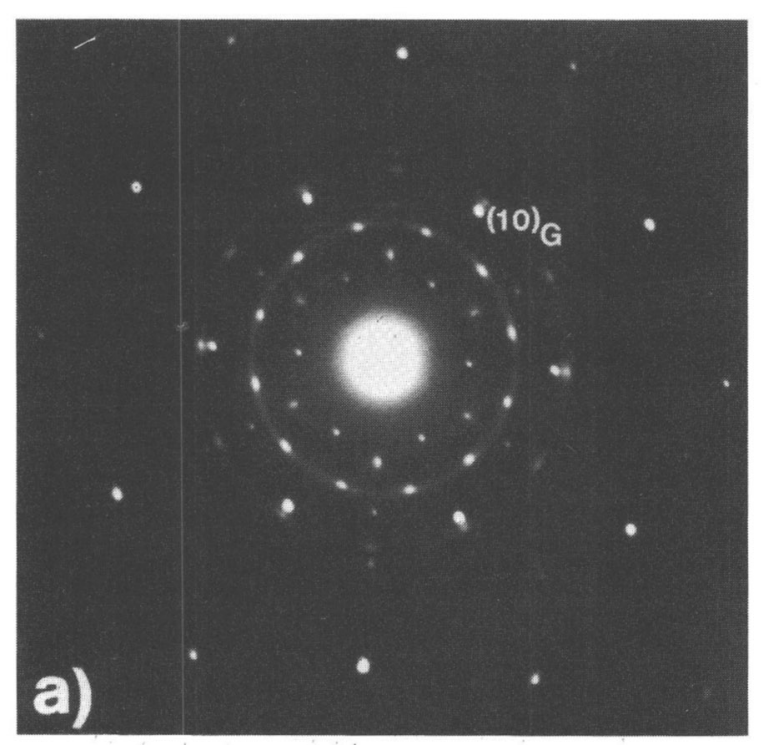

$\odot$

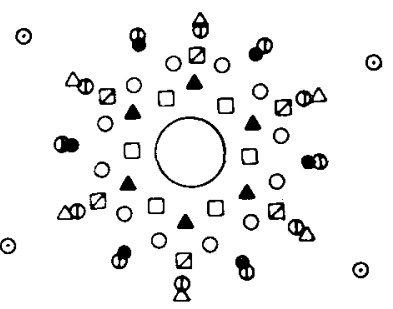

c)

$$
\begin{aligned}
& \text { - }(10)_{G} \square(10)_{2 \times 2} \Delta(10)_{\sqrt{3} \times \sqrt{3}} \\
& \odot(11)_{G} \nabla(11)_{2 \times 2} \Delta(20)_{\sqrt{3}} \times \sqrt{3}
\end{aligned}
$$

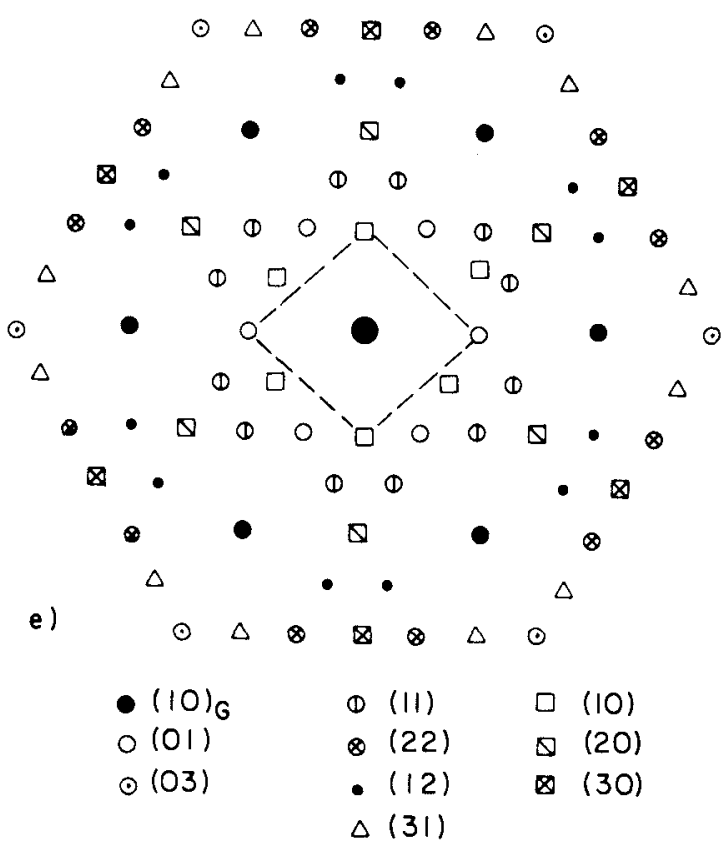

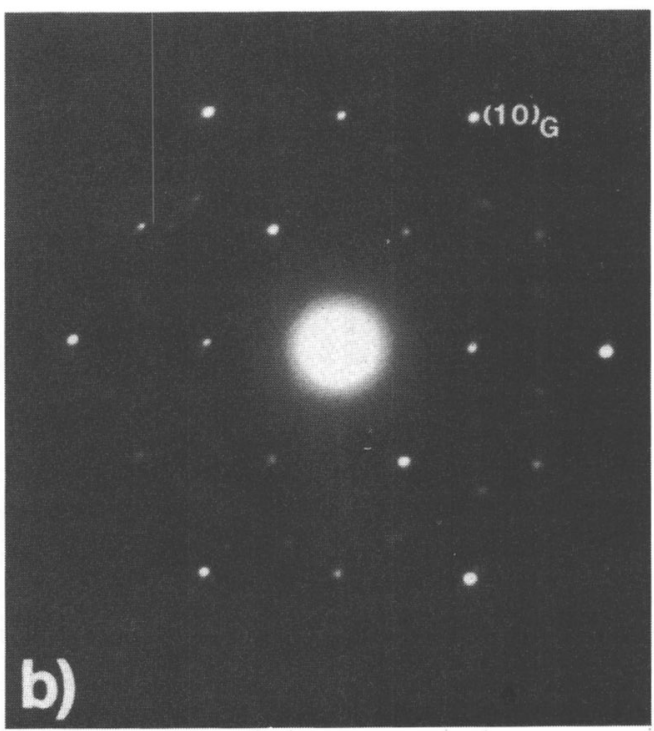

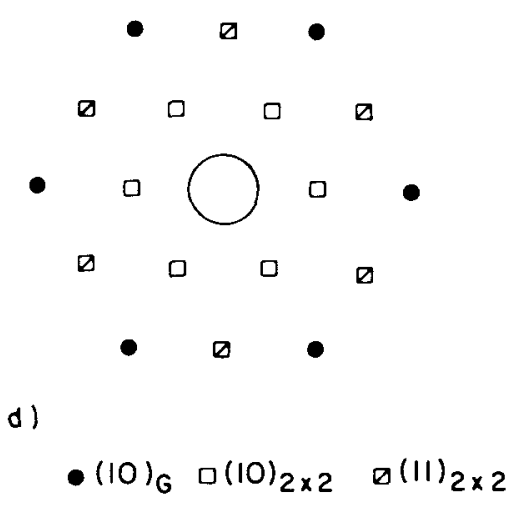

FIG. 6. The $(h k 0)$ electron diffraction patterns of HOPG intercalated with $\mathrm{KH}$ at: (a) $290^{\circ} \mathrm{C}$ and (b) $430^{\circ} \mathrm{C}$. For (a) superlattice spots for the commensurate $(2 \times 2) R 0^{\circ}$ and $(\sqrt{3} \times \sqrt{3}) R 30^{\circ}$ structures are observed. In addition, elongated spots and a thin ring corresponding to wave vectors $q=2.21 \AA^{-1}$ and $q=3.11 \AA^{-1}$ are identified (see text). For (b) the dominant superlattice spots correspond to the commensurate $(2 \times 2) R 0^{\circ}$ structure. Schematic diagrams are given for (a) in (c) and for (b) in (d), respectively, and for a $(2 \times \sqrt{3}) R\left(0^{\circ}, 30^{\circ}\right)$ commensurate phase in (e). (e) shows the superposition of the three equivalent domains; the reciprocal space unit cell for one of the domains is shown with a dashed line. 
intercalation process. The TEM results suggest that the intermediate phase between a potassium-GIC and a $\mathrm{KH}_{x}$-GIC is that observed at the "boundary" regions and, further, that as the intercalation proceeds, the boundary moves toward the pure potassium-GIC region. The net effect is that the hydrogen saturated regions grow at the expense of the pure potassium regions as the intercalation proceeds.

The hydrogen deficient regions at the boundary between pure potassium regions and regions with normal hydrogen concentration are different for stages 1 and 2, probably because for the stage 1 potassiumGIC's, the interaction between neighboring intercalate layers is stronger than for the stage 2 potassium-GIC's. Thus when the intercalation of hydrogen starts, the hydrogen atoms are more likely to form layers of $\mathrm{KH}$ that are separated from each other, and only every other intercalate layer contains hydrogen. As the intercalation of hydrogen proceeds, the hydrogen goes in the remaining potassium layers until all the intercalate layers contain hydrogen. On the other hand, for the stage 2 potassium-GIC samples the interaction between neighboring intercalate layers is weaker so that the hydrogen atoms more readily go into every intercalate layer.

Other values for repeat distances were obtained using the TEM in other regions of certain stage 1 samples: $8.07 \pm 0.08 \AA$ and $9.36 \pm 0.08 \AA$. A repeat distance of $\sim 9.40 \AA$ was also obtained from $(00 l)$ lattice fringes from desorbed areas of fibers intercalated with $\mathrm{KH}$ by the chemical absorption method as explained below. These $I_{c}$ values are perhaps related to partially desorbed regions, where hydrogen deficient phases might exist. It is believed that for very low hydrogen uptake, the intercalate consists of a single layer of $K$ atoms and the hydrogen atoms go into vacancies in the potassium layer. The KHK sandwich is initiated ${ }^{3}$ for $x>0.1$. Such layers of lower hydrogen density $(0.1<x \ll 0.66)$ may correspond to lower $I_{c}$ values.

We have also studied the in-plane structure of $\mathrm{KH}_{x}-\mathrm{GIC}$ 's using the TEM for several intercalation temperatures and times. Two commensurate in-plane phases were found to coexist in samples intercalated with $\mathrm{KH}$ (or KD) for stages 1 and 2 and intercalation temperatures between $200^{\circ} \mathrm{C}$ and $430^{\circ} \mathrm{C}: \mathrm{a}(2 \times 2) R 0^{\circ}$ phase and a $(\sqrt{3} \times \sqrt{3}) R 30^{\circ}$ phase [see Fig. $\left.6(\mathrm{a})\right] .{ }^{15}$ In addition, a set of spots is found at $2.21 \AA^{-1}$ and 3.11 $\AA^{-1}$, as seen in Fig. 6(a), corresponding to an incommensurate phase with reciprocal lattice vectors in agreement with the (200) and (220) reciprocal lattice vectors for pristine $\mathrm{KH}$. Figure 6(c) shows a schematic diagram for the commensurate $\mathrm{KH}_{x}-$ GIC phases and the graphite spots observed in Fig. 6(a). The sets of spots at $2.21 \AA^{-1}$ and $3.11 \AA^{-1}$ are found as the difference between Figs. 6(a) and 6(c). During the TEM observation, several changes in the diffraction pattern take place; the commensurate phases weaken in intensity and finally disappear, while the sets of spots at 2.21 $\AA^{-1}$ and $3.11 \AA^{-1}$ become more intense and finally become sharp rings. To minimize the effect of the electron beam, an accelerating voltage of $100 \mathrm{kV}$ was used, and even with this lower voltage, spots at $2.21 \AA^{-1}$ and 3.11 $\AA^{-1}$ corresponding to pristine $\mathrm{KH}$ were observed. It is possible that these elongated incommensurate spots correspond to small crystallites of $\mathrm{KH}$ sitting on the surface of the sample due to desorption primarily during the TEM observation.

The elongated (200) and (220) spot patterns at $q=2.21 \AA^{-1}$ and $3.11 \AA^{-1}$, respectively, are identified with an orientational alignment of the unit vectors of the $\mathrm{KH}$ crystallites with those of the graphite substrate due to the epitaxial growth of the KH crystallites. It has been reported ${ }^{9}$ that for $\mathrm{NaH}_{x}-$ GIC's, some of the intercalate retains its pristine hydride form and is probably located in inclusions between the graphite layers. It is also possible that some small $\mathrm{KH}$ crystallites are present in the $\mathrm{KH}_{x}-\mathrm{GIC}$ samples. The TEM-induced desorption occurs since an accelerating voltage of $100 \mathrm{kV}$ gives the electrons a kinetic energy above the threshold energy for atom displacements in knock-on collisions with the hydrogen atoms. ${ }^{16}$ We believe that the rings at 2.21 $\AA^{-1}$ and $3.11 \AA^{-1}$ observed in the electron diffraction patterns after electron beam irradiation are primarily due to speckles of desorbed $\mathrm{KH}$ on the surface of the sample.

Figure 6(b) shows an electron diffraction pattern of a stage 1 sample intercalated with $\mathrm{KH}$ at $430^{\circ} \mathrm{C}$. This figure shows only the $(2 \times 2) R 0^{\circ}$ commensurate inplane structure and very weak spots at the $2.21 \AA^{-1}$ and $3.11 \AA^{-1}$ reciprocal lattice vectors. Figure 6(d) gives a schematic for the $(2 \times 2) R 0^{\circ}$ phase shown in Fig. 6(b). Some electron diffraction patterns obtained from other regions of the same sample showed weak $(\sqrt{3} \times \sqrt{3}) R 30^{\circ}$ spots indicating a small admixture of the two phases. This result indicates that the higher intercalation temperature of $430^{\circ} \mathrm{C}$ favors the formation of the less dense $(2 \times 2) R 0^{\circ}$ in-plane phase.

Figure 7 shows dark field images using the $(2 \times 2) R 0^{\circ}$ [Fig. 7(a) ] and $(\sqrt{3} \times \sqrt{3}) R 30^{\circ}[$ Fig. 7 (b) ] beams taken from a region of the sample intercalated at $430^{\circ} \mathrm{C}$ where the two in-plane phases coexisted. These dark field images show small $(\sim 250 \AA)$ islands of the $(\sqrt{3} \times \sqrt{3}) R 30^{\circ}$ phase [Fig. 7 (b)] separated by a large $(\sim 1000 \AA)$ background of the $(2 \times 2) R 0^{\circ}$ phase [Fig. 7 (a) ]. This figure indicates that the two commensurate in-plane structures form separate phases.

Dark field images obtained from the $(\sqrt{3} \times \sqrt{3}) R 30^{\circ}$ and the $(2 \times 2) R 0^{\circ}$ spots of a sample intercalated at $320^{\circ} \mathrm{C}$ showed islands of dimension of $\sim 300 \AA$ for the $(\sqrt{3} \times \sqrt{3}) R 30^{\circ}$ phase and $\sim 600 \AA$ for the $(2 \times 2) R 0^{\circ}$ phase. Analysis of dark field images from the two in- 

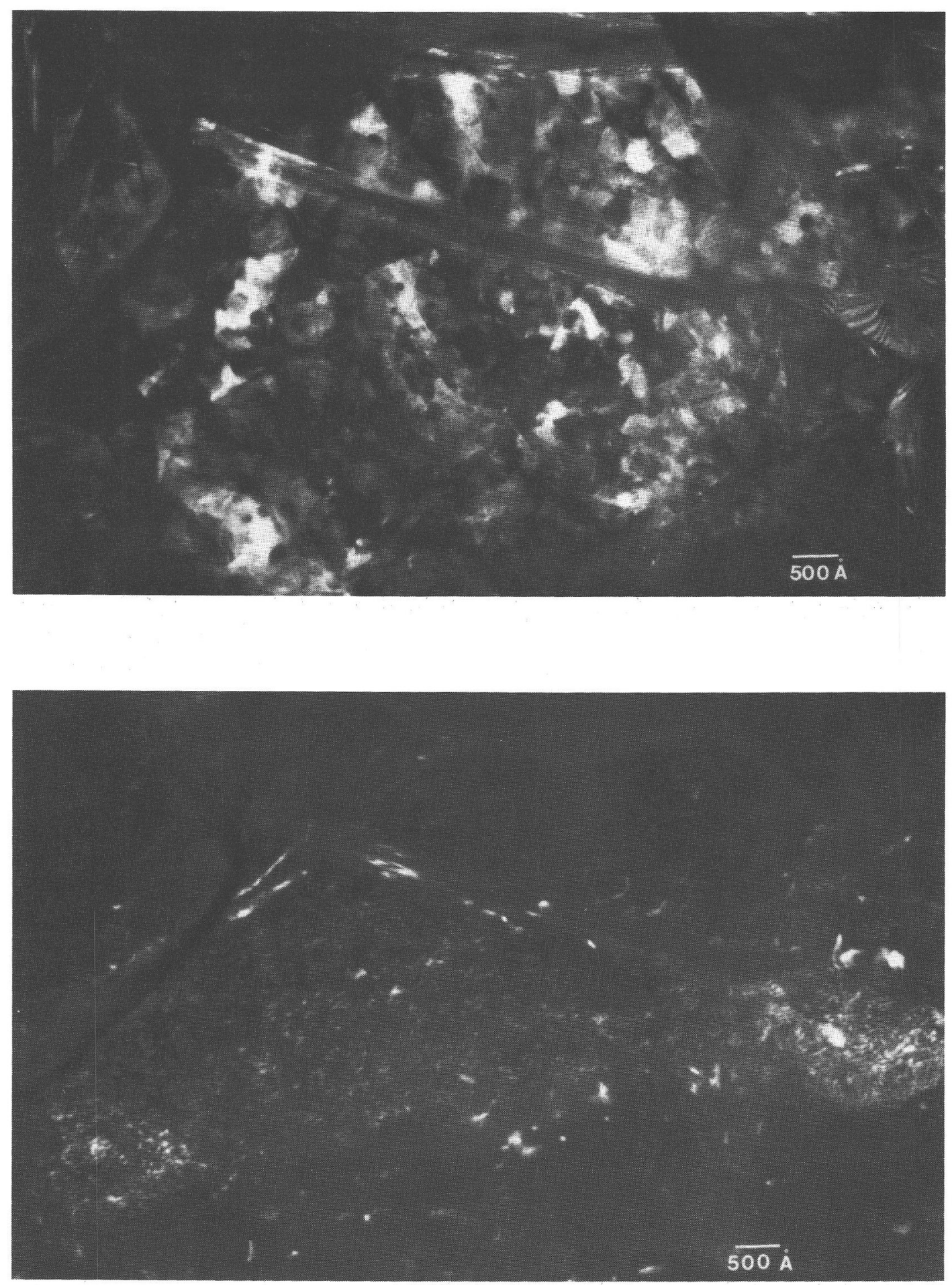

FIG. 7. Dark field images of a sample intercalated with $\mathrm{KH}$ at $430^{\circ} \mathrm{C}$ using the direct intercalation process. The images were obtained using the (100) diffraction beams of (a) the $(2 \times 2) R 0^{\circ}$ and (b) the $(\sqrt{3} \times \sqrt{3}) R 30^{\circ}$ commensurate phases. The images in (a) and (b) were obtained from the same region of the sample.

plane phases for samples intercalated at different temperatures showed that the relative concentration of the two phases depends on intercalation temperature. Our results indicate that the $(2 \times 2) R 0^{\circ}$ phase is dominant for high intercalation temperatures whereas the $(\sqrt{3} \times \sqrt{3}) R 30^{\circ}$ phase is more pronounced for low intercalation temperatures. Similar $(h k 0)$ electron diffraction patterns and $c$ axis repeat distances were obtained 
for the $\mathrm{KD}_{y}$-GIC samples. This indicates that both intercalants form the same structure upon intercalation, as was expected from their structure in the pristine form. It is interesting to note that these two in-plane phases have also been observed in KHg-GIC's. ${ }^{12,13}$ In the $\mathrm{KHg}$-GIC system, a $(2 \times \sqrt{3}) R\left(0^{\circ}, 30^{\circ}\right)$ commensurate phase has also been observed using the TEM. ${ }^{12}$ The $(2 \times \sqrt{3}) R\left(0^{\circ}, 30^{\circ}\right)$ phase is schematically presented in Fig. 6(e). The chemical formula $\mathrm{C}_{4 n} \mathrm{KH}_{x}$, where $n$ is the stage index suggested by Guérard, suggests a $(2 \times 2) R 0^{\circ}$ structure or also a $(2 \times \sqrt{3}) R\left(0^{\circ}, 30^{\circ}\right)$ structure. Up to now we have not identified this phase in the $\mathrm{KH}_{x}$ - GIC system.

A possible arrangement for the $\mathrm{K}$ and $\mathrm{H}$ atoms in the $(\sqrt{3} \times \sqrt{3}) R 30^{\circ}$ phase and the $(2 \times 2) R 0^{\circ}$ phase is the placement of $\mathrm{K}$ atoms at either the $(\sqrt{3} \times \sqrt{3}) R 30^{\circ}$ sites in an $\alpha \alpha$ stacking or at the $(2 \times 2) R 0^{\circ}$ sites in either an $\alpha \alpha$ or $\alpha \beta$ stacking sequence within the intercalate sandwich. In the $\alpha \alpha$ stacking, the two $\mathrm{K}$ atoms in the intercalate sandwich sit on top of each other and in the $\alpha \beta$ stacking they sit in equivalent but distinctly different positions while the hydrogen atoms are at equivalent positions that are not occupied by the $\mathbf{K}$ atoms. For the $(2 \times 2) R 0^{\circ}$ commensurate phase in this model, the graphite bounding layers have an AA stacking when the potassium atoms within the intercalate layer have an $\alpha \alpha$ stacking and an $\mathrm{AB}$ stacking when the potassium atoms have an $\alpha \beta$ stacking. It should be noted that the $\alpha \beta$ stacking could yield a smaller $I_{c}$ value, because of the tighter spatial packing. An $\alpha \beta$ stacking of the $\mathrm{K}$ atoms within the intercalate sandwich in the $(\sqrt{3} \times \sqrt{3}) R 30^{\circ}$ phase, does not leave enough sites available for the hydrogen atoms except for low concentrations of hydrogen. Therefore we believe that for the $(\sqrt{3} \times \sqrt{3}) R 30^{\circ}$ phase, it is more likely that the two potassium atoms within the intercalate sandwich arrange themselves in an $\alpha \alpha$ stacking sequence. It is also possible that the $(\sqrt{3} \times \sqrt{3}) R 30^{\circ}$ phase corresponds to a region with lower hydrogen content.

A possible structure for the $\mathrm{KH}_{x}$-GIC system has been suggested on the basis of neutron scattering experiments from $\mathrm{C}_{8} \mathrm{KH}_{2 / 3}$ samples. ${ }^{17}$ The proposed mod$\mathrm{el}^{17}$ consists of a sandwich of two $(2 \times 2) R 0^{\circ}$ layers of $\mathrm{K}$ atoms placed face to face and shifted by $a_{0}$ (the graphite lattice constant) with respect to one another. In this model the hydrogen atoms take the unoccupied sites. Thus for a small value of $x \sim 0.66$, not all the hydrogen sites are occupied and the unit cell is larger than the $(2 \times 2) R 0^{\circ}$ unit cell. An orthorhombic unit cell with parameters $a=8.56 \AA, b=12.39 \AA$, and $c=2 I_{c}=17.06$ $\AA$ has also been suggested for the $\mathrm{KH}_{x}$-GIC system. ${ }^{2}$ Up to now we have no TEM evidence for this large spa-

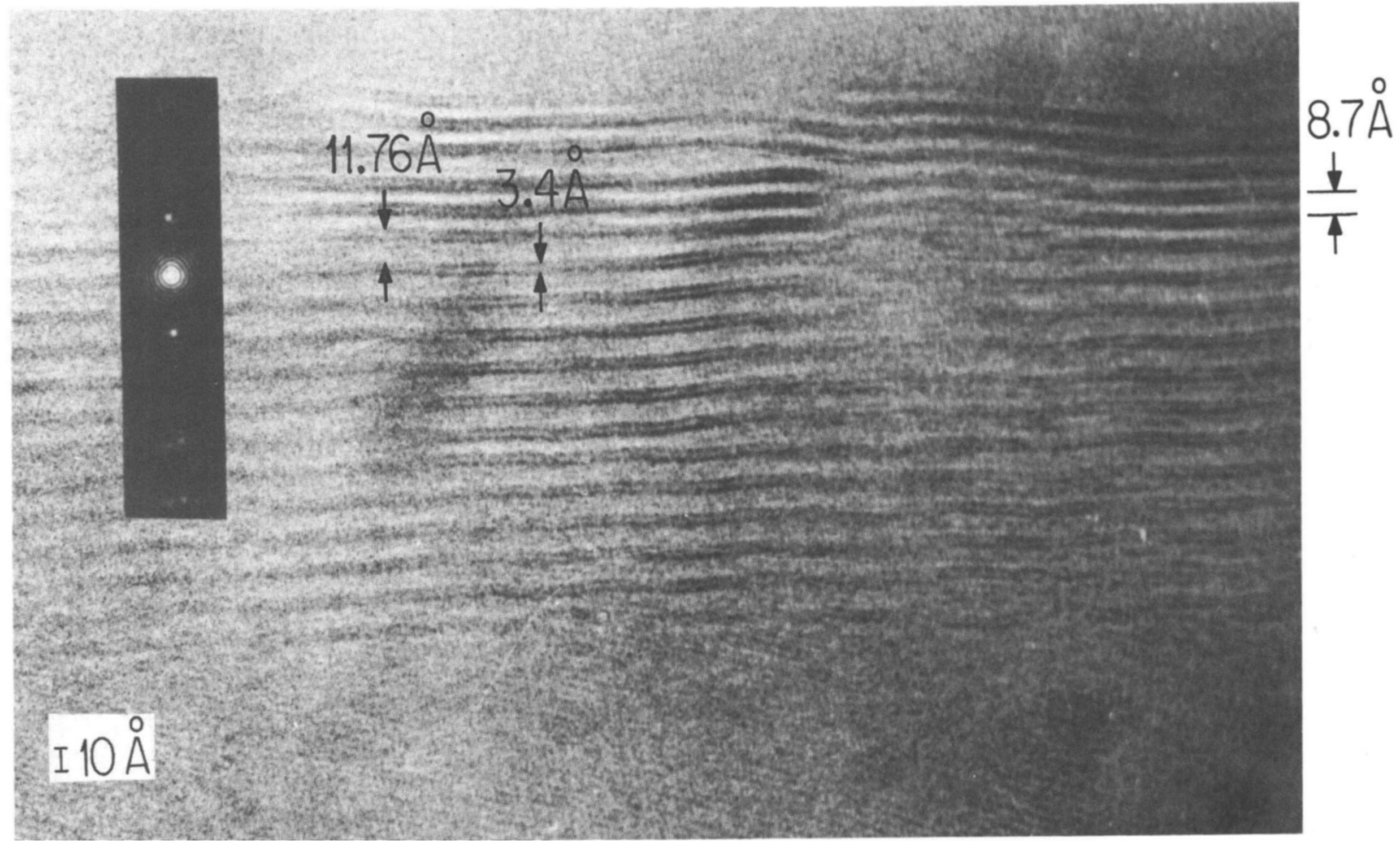

FIG. 8. Bright field $c$-axis lattice fringes of a stage 2 intercalated fiber (BDGF) prepared by chemical absorption of hydrogen into a stage $1 \mathrm{C}_{8} \mathrm{~K}$ in tercalated fiber. The figure shows a $\mathrm{C}_{8} \mathrm{KH}_{x}$ region and $\mathrm{C}_{24} \mathrm{~K}$ (desorbed) region. The inset is an optical diffractogram taken from the negative of the photograph. The chemical formulas for the stage 1 and stage $2 \mathrm{~K}$-GIC's and stage $2 \mathrm{KH}_{x}-$ GIC are taken from Ref. 2. 
tial periodicity. It should be noted that the TEM is more sensitive to the $\mathrm{K}$ atoms than to the hydrogen atoms, and according to Trewern et al., ${ }^{17}$ it is the hydrogen atoms that define the larger unit cell. Quasielastic neutron scattering experiments on stage $2 \mathrm{KH}_{x}-\mathrm{GIC}\left(\mathrm{KD}_{y-}\right.$ -GIC) samples prepared by the chemical absorption method, showed no evidence for hydrogen (deuterium) motion for momentum transfers up to $2.5 \AA^{-1}$ (resolution $\sim 100 \mu \mathrm{eV}$ at room temperature and $\sim 1 \mu \mathrm{eV}$ at 453 K). ${ }^{18}$

We have also studied the structure of fibers (BDGF) intercalated with $\mathrm{KH}$ and $\mathrm{KD}$ using the chemical absorption method. ${ }^{3,4}$ Figure 8 shows $c$-axis fringes of a stage $2 \mathrm{KH}_{x}$ intercalated fiber prepared by chemical absorption of hydrogen into a stage 1 potassium intercalated fiber. This figure shows two different repeat distances in two separate regions of the fiber. In one of these regions a repeat distance of $I_{c} \sim 11.76 \pm 0.08 \AA$ was observed corresponding to the stage $2 \mathrm{KH}_{x}$-GIC in agreement with the value of 11.88 $\AA$ reported by Guérard et al. ${ }^{4}$ In this region, fringes with spacing $\sim 3.4 \AA$ periodically stacked between layered structures with spacings $\sim 8.4 \AA$ are clearly observed, demonstrating the structure of the stage 2 $\mathrm{KH}_{x}-\mathrm{GIC}$ with $I_{c}=11.8 \AA$. Also in this figure, a repeat distance of $\sim 8.7 \AA$ can be observed in a different region of the sample. This repeat distance is in agreement with the value reported for stage 2 potassium$-\mathrm{GIC}^{1}$ and corresponds to a desorbed region of the sample. It is important to note that the texture observed in the desorbed region is different from that of potassium compounds that have been directly synthesized using BDGF as host material. ${ }^{19}$ The region in the boundary between the $\mathrm{KH}_{x}-\mathrm{GIC}$ regions corresponds to a hydrogen deficient stage 2 with a repeat distance of $\sim 9.40 \AA$. This repeat distance was also observed in some regions of the HOPG samples prepared by the direct intercalation method. This result suggests that the desorbed compound is similar for both intercalation processes. The smaller repeat distance $(11.76 \AA)$ observed for samples synthesized using the chemical absorption method than that for samples synthesized by the direct intercalation method (11.90-12.08 $\AA$ ) is consistent with a smaller hydrogen content $(x \sim 0.66)$ for samples prepared by the chemical absorption method ${ }^{4}$ than for the direct intercalation method ${ }^{2}(x \sim 0.8)$.

The high degree of in-plane structural order observed in $\mathrm{KH}_{x}$-GIC's [see, for example, Fig. 6(b)] ranks them among the best ordered intercalation compounds that can be synthesized. In addition to this high degree of structural order, the ionic nature of $\mathrm{KH}$ creates high interest in the charge transfer associated with these compounds $5,11,20$

\section{ACKNOWLEDGMENTS}

We wish to thank Dr. G. Dresselhaus for many valuable discussions.

The work by L.S.-R., N.C.Y., and M.S.D. was supported by Air Force Office of Scientific Research Contract No. F49620-83-C0011 and the work by M.E. and T.E. was supported by Department of Energy Contract No. DE-AC02-83ER45041.

\section{REFERENCES}

${ }^{1}$ M. S. Dresselhaus and G. Dresselhaus, Adv. Phys. 30, 139 (1981).

${ }^{2}$ D. Guérard, C. Takoudjou, and F. Rousseaux, Synth. Met. 7, 43 (1983).

${ }^{3}$ M. Colin and A. Hérold, Bull. Soc. Chim. Fr., 1982 (1971).

${ }^{4}$ D. Guérard, P. Lagrange, and A. Hérold, Mater. Sci. Eng. 31, 29 (1977).

${ }^{5}$ N. C. Yeh, T. Enoki, L. E. McNeil, G. Roth, L. Salamanca-Riba, M Endo, and G. Dresselhaus, MRS Extended Abstracts, Graphite Intercalation Compounds, edited by P. C. Eklund, M. S. Dresselhaus, and G. Dresselhaus (Materials Research Society, Pittsburgh, PA, 1984), p. 246.

${ }^{6} \mathrm{~L}$. Salamanca-Riba, Ph.D, thesis, Massachusetts Institute of Technology, Cambridge, MA, 1985.

7J. C. H. Spence, Experimental High Resolution Electron Microscopy (Clarendon, Oxford, 1981).

${ }^{8}$ N. C. Yeh, T. Enoki, L. Salamanca-Riba, and G. Dresselhaus, Extended Abstracts of the 17th Biennial Conference on Carbon, Lexington, June 1985, p. 194.

${ }^{9}$ D. Guérard, N. E. Elalem, C. Takoudjou, and F. Rousseaux, Synth. Met. 12, 195 (1985).

${ }^{10} \mathrm{G}$. L. Doll and P. C. Eklund (to be submitted for publication).

${ }^{11}$ T. Enoki, N. C. Yeh, S. T. Chen, and M. S. Dresselhaus, Phys. Rev. B 33, 1292 (1986).

${ }^{12} \mathrm{G}$. Timp, Ph.D. thesis, Massachusetts Institute of Technology, Cambridge, MA, 1983.

${ }^{13}$ M. El Makrini, P. Lagrange, D. Guérard, and A. Hérold, Carbon 18, 211 (1980).

${ }^{14} \mathrm{P}$. Lagrange, M. El Makrini, and A. Hérold, Rev. Chim. Minér. 20, 229 (1983).

${ }^{15}$ L. Salamanca-Riba, N. C. Yeh, T. Enoki, M. S. Dresselhaus, and M. Endo, in Ref. 5, p. 249.

${ }^{16} \mathrm{~L}$. W. Hobbs, in Proceedings of the 25 th Scottish University Summer School in Physics, Glasgow, August 1983, edited by J. N. Chapman and A. J. Craven (SUSSP, Edinburgh, 1983), p. 399.

${ }^{17}$ T. Trewern, R. K. Thomas, G. Naylor, and J. W. White, J. Chem. Soc., Faraday Trans. 178, 2369 (1982).

${ }^{18} \mathrm{~T}$. Trewern, R. K. Thomas, and J. W. White, J. Chem. Soc., Faraday Trans. 1 78, 2399 (1982).

${ }^{19}$ M. Endo, T. C. Chieu, G. Timp, M. S. Dresselhaus, and B. S. Elman, Phys. Rev. B 28, 6982 (1983).

${ }^{20} \mathrm{~T}$. Enoki, H. Inokuchi, and M. Sano, in Ref. 5, p. 243. 